\title{
Stroke risk associated with balloon based catheter ablation for atrial fibrillation: Rationale and design of the MACPAF Study
}

Karl Georg Haeusler ${ }^{1,2^{*}+}$, Lydia Koch ${ }^{3 \dagger}$, Juliane Ueberreiter ${ }^{1}$, Matthias Endres ${ }^{1,2}$, Heinz-Peter Schultheiss ${ }^{3}$, Peter U Heuschmann ${ }^{2}$, Alexander Schirdewan ${ }^{3}$, Jochen B Fiebach ${ }^{2}$

\begin{abstract}
Background: Catheter ablation of the pulmonary veins has become accepted as a standard therapeutic approach for symptomatic paroxysmal atrial fibrillation (AF). However, there is some evidence for an ablation associated (silent) stroke risk, lowering the hope to limit the stroke risk by restoration of rhythm over rate control in AF. The purpose of the prospective randomized single-center study "Mesh Ablator versus Cryoballoon Pulmonary Vein Ablation of Symptomatic Paroxysmal Atrial Fibrillation" (MACPAF) is to compare the efficacy and safety of two balloon based pulmonary vein ablation systems in patients with symptomatic paroxysmal AF.
\end{abstract}

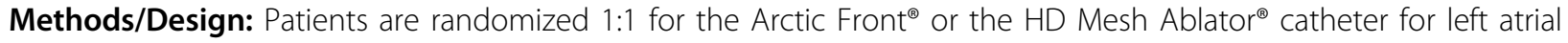
catheter ablation (LACA). The predefined endpoints will be assessed by brain magnetic resonance imaging (MRI), neuro(psycho)logical tests and a subcutaneously implanted reveal recorder for AF detection. According to statistics 108 patients will be enrolled.

Discussion: Findings from the MACPAF trial will help to balance the benefits and risks of LACA for symptomatic paroxysmal AF. Using serial brain MRIs might help to identify patients at risk for LACA-associated cerebral thromboembolism. Potential limitations of the study are the single-center design, the existence of a variety of LACA-catheters, the missing placebo-group and the impossibility to assess the primary endpoint in a blinded fashion,

Trial registration: clinicaltrials.gov NCT01061931

\section{Background}

About $20-37 \%$ of all ischemic strokes are of cardioembolic origin [1,2], caused by different cardiac disorders, but most commonly by atrial fibrillation (AF). The current demographic trends will lead to an increasing prevalence of AF $[3,4]$ and implicate a growing risk of cardioembolic stroke [1]. In general, cardioembolic stroke has a worse prognosis and a higher recurrence rate than other ischemic stroke subtypes [5]. According to recent guidelines, the left atrial catheter ablation (LACA) of pulmonary veins (PV) is approved for treatment of symptomatic atrial fibrillation (AF) in patients with paroxysmal or persistent AF, refractory to

\footnotetext{
* Correspondence: georg.haeusler@charite.de

+ Contributed equally

'Department of Neurology, Charité - University Medicine Berlin, Germany
}

antiarrhythmic medication [6]. Within the last years, LACA using radiofrequency has become a routine procedure and is widely used [7]. In patients with paroxysmal AF ( $<65$ years) and without structural heart disease the achieved rate of persisting sinus rhythm is best and varies between $60 \%$ and $80 \%$ [8,9]. Major complications like pericardial tamponade, $\mathrm{PV}$-stenosis, atrial-esophageal fistula, injury of the phrenic nerve and vascular damage by atrial and venous sheaths were detected more often in older patients ( $\geq 75$ years) and patients with congestive heart failure [10]. Furthermore, there is some evidence for an ablation associated stroke risk $[9,11,12]$, lowering the (so far) unproven hope to limit the AF-associated stroke risk by LACA. Considering that most AF patients "eligible" for LACA have a low stroke risk [13], the evident stroke rate of $0.4-1.1 \%$ in
C Biomed Central

() 2010 Haeusler et al; licensee BioMed Central Ltd. This is an Open Access article distributed under the terms of the Creative Commons Attribution License (http://creativecommons.org/licenses/by/2.0), which permits unrestricted use, distribution, and reproduction in any medium, provided the original work is properly cited. 
several registries [9-12] and a "silent" stroke rate of 10$11.3 \%[14,15]$ raise concerns and afford further investigation.

As demonstrated in humans, a balloon catheter using cryoenergy (Arctic Front ${ }^{\odot}$, Medtronic, Inc.) had safety advantages compared to radiofrequency devices [16]. Another basket shaped catheter device (HD Mesh ablator ${ }^{\oplus}$, C.R. Bard, Inc.) combines circumferential mapping and radiofrequency delivery, leading to a reduced procedure and fluoroscopy time [17]. Both catheters are already used in daily clinical practice at the Department of Cardiology, Charité Campus Benjamin Franklin (CBF). Therefore, we designed a randomized single center study to evaluate the risks and benefits of LACA, using these innovative catheter devices.

Conducting MACPAF, we focus on the devices' periprocedural (silent) stroke rates and subsequent neuro (psycho)logical deficits. By using a highly standardized monitoring including serial brain magnetic resonance imaging (MRI) and neuro(psycho)logical tests we hope to set new standards for further trials in this regard. Furthermore, the implanted reveal recorder Reveal $\mathrm{XT}^{\odot}$ (Medtronic, Inc.) is highly accurate to detect clinically silent AF episodes, not infrequently occurring after LACA [18] on the one hand, and to determine the AF burden, on the other hand.

\section{Methods/Design}

\section{Study population and study design}

The Mesh Ablator versus Cryoballoon Pulmonary Vein Ablation of Symptomatic Paroxysmal Atrial Fibrillation (MACPAF) study is designed as a prospective randomized single center study conducted by the Department of Cardiology and Pneumology (Charité, CBF), the Center for Stroke Research Berlin and the Department of Neurology (Charité, CBF). The study has been approved by the local Ethics Committee (EA4/087/08) in February 2009. Randomization started in April 2009. Study entry and storage of data require subsequent consent by the patient. The inclusion and exclusion criteria are summarized in Table 1. Before undergoing LACA, patients receive an implantable device (Reveal $\mathrm{XT}^{\oplus}$, Medtronic, Inc.) able to record the AF burden for at least 2 years. Within $24 \mathrm{~h}$ before LACA, all patients undergo transesophageal echocardiography (for exclusion of atrial thrombi), a brain MRI as well as a neuro(psycho)logical examination. A repeat brain MRI and neuro(psycho) logical examination will be performed within $48 \mathrm{~h}$ after the procedure. Patients will be randomized 1:1 for the cryoenergy device (Arctic Front ${ }^{\oplus}$ ) or the newest device using conventional radiofrequency (HD Mesh ablator ${ }^{\oplus}$ ). Before PV ablation, a CT image will be performed and segmented by CARTO Merge ${ }^{\oplus}$ (Biosense Webster, Inc.) for visualization of PV anatomy. The LACA procedure
Table 1 MACPAF: Inclusion and exclusion criteria

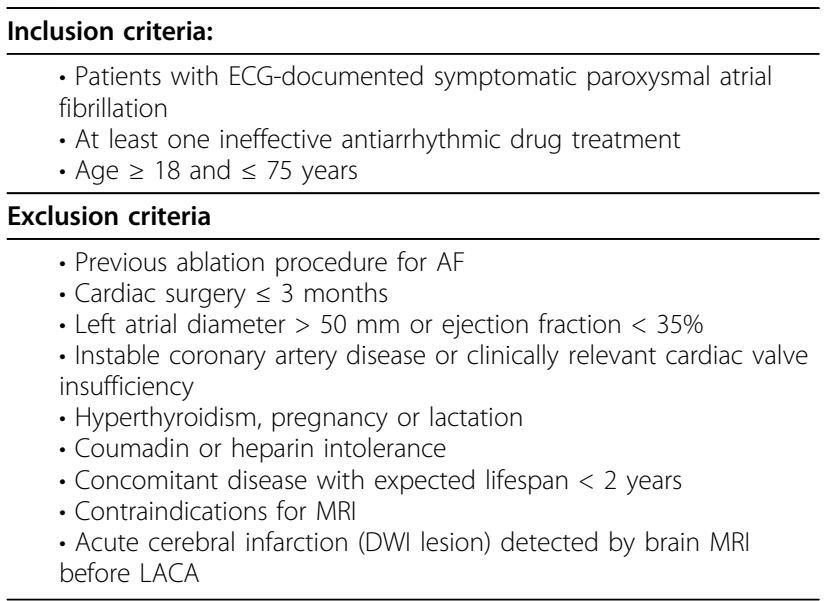

will be performed by first introducing a ring shaped decapolar catheter via a single transseptal puncture. Afterwards, this catheter will be exchanged for the cryoballoon catheter or the mesh ablator, respectively. Finally, PV conduction will be re-evaluated by the decapolar catheter. In case of remaining PV conduction, the ablation procedure will be continued until isolation of all PVs is achieved. After transseptal puncture, heparin will be given to assure an activated clotting time of $>300$ s. Follow-ups will take place at 1, 3, 6, 9 and 12 months after LACA, including data acquisition from the Reveal $\mathrm{XT}^{\oplus}$ or a 5 -day-holter-ECG, respectively. Brain MRI and neuro(psycho)logical examination will be repeated after 6 months. The patient will be put on oral anticoagulation (INR 2-3) for at least 6 months after LACA to improve comparability of the results. The end of study is expected for late 2011.

\section{Aims and objectives}

The primary objective of MACPAF is the efficacy of PV isolation, defined by achieving an exit block for all PVs per patient. We hypothesize that the cryoballoon catheter is more effective compared to the mesh ablator catheter in this regard. This is the only objective, which can not be assessed in a blinded fashion for the used ablation catheter. The secondary objective is the detection of LACA-associated (silent) cerebral thromboembolism by brain MRI and by neuro(psycho)logical examination. Further objectives are the determination of the AF recurrence rate after LACA using an implantable device, the detection of non-neurological major complications after LACA and the detection of (silent) cerebral thromboembolism within 6 months after LACA.

\section{Sample size calculation and Statistical analysis}

The primary endpoint for a patient is achieved when all four pulmonary veins are isolated. We hypothesize that 
corresponding to this primary endpoint the Arctic Front $^{\oplus}$ catheter is superior to the HD Mesh Ablator $^{\oplus}$ catheter. The null hypothesis of equal proportions of successful outcomes for both methods will be tested by the two-sided Fisher's exact test at significance level 0.05 . Assuming a proportion of $75 \%$ successful ablations for the HD Mesh Ablation ${ }^{\circ}$ catheter, sample sizes of 54 for both treatment groups achieve $80 \%$ power to detect a $20 \%$ greater proportion of successes for the Arctic Front $^{\circ}$ catheter. This corresponds to a proportion of 95\% successful ablations for the Arctic Front ${ }^{\circ}$ catheter a relative improvement of $27 \%$ for the Arctic Front ${ }^{\circ}$ catheter in comparison to the HD Mesh Ablator ${ }^{\circ}$ catheter.

An intention-to-treat analysis will be used to analyze treatment differences concerning predefined outcome measures. Treatment allocation will be done by genderstratified randomization. For all outcome variables descriptive statistics will be collected. For quantitative traits, we will compute absolute and relative frequencies. In the case of continuous or quasi-continuous variables with nearly symmetric distribution, we will compute arithmetic mean, standard deviation, minimal and maximal values, otherwise median, quartiles, minimal and maximal values. Fisher's exact test will be used to compare proportions for dichotomous outcomes between independent groups or to test independency of two dichotomous traits within a population. Hypotheses about proportions for dichotomous outcomes within a single group (one sample analysis) will be tested by the binomial test. Assuming equal shaped distributions in the two treatment groups for at least ordinal scaled outcome variables group specific location parameters will be compared by the t-test or the Mann-Whitney test, respectively, depending on normality or non-normality of the distribution. Analysis regarding time to first AFrecurrence: Defining 'no observed AF-recurrence between 3 months after LACA and the end of followup' as 'survival', survival functions will be estimated by the Kaplan-Meier method. The null hypothesis of equal survival curves for the two treatment groups will be tested by the Log-Rank test.

\section{MRI analysis}

MRI examination is performed if the patient is able to give informed consent. Using a 3T MR scanner (Tim Trio; Siemens AG, Erlangen, Germany) the following sequences will be done 24 hours before as well as 24-48 hours after the ablation procedure: $\mathrm{T} 2$ *-weighted imaging to screen for intracranial hemorrhage; diffusionweighted magnetic resonance imaging (DWI) to assess cerebral infarction; Fluid-attenuated inverse recovery (FLAIR) to estimate microangiopathic lesions load and to investigate the age of recent lesions; time-of-flight
MR-Angiography (TOF MRA) to detect vessel stenosis or occlusion. Six month after LACA a third measurement without TOF MRA will be performed to assess the incidence of (silent) strokes by time. For sequence parameters see [19]. Blinded MRI reading will be done by a board certified neuroradiologist (JBF).

\section{Neurological examination}

The National Institute of Health Stroke Scale (NIHSS) is a validated instrument used in many clinical trials to assess neurological deficits of stroke patients [20]. The NIHSS displays the subjects' consciousness, the ability to move limbs, the sensorium, speech, vision and eye movements. The modified Rankin Scale (mRS) is used to quantify the persisting handicap after stroke [21].

\section{Neuropsychological examination}

The neuropsychological assessment was designed to assess important cognitive domains and includes the following tests: Trail Making Test A and B (information processing and divided attention), the Stroop Test, the Category and Letter Fluency Test (all three assessing different aspects of executive functions) [22], the subtest 3 from the German Leistungsprüfsystem (reasoning) [23], a German version of the Rey Auditory Verbal Learning Test [24], the digit-span forward and backward test from the Wechsler Memory Scale - Revised [22] (verbal short-term and working memory), the German Mehrfachwahl-Wortschatz-Intelligenztest (MWT-A Test; educational level) [25] as well as the Rey-Osterrieth Complex Figure Test (visuo-spatial skills and visual memory) [22].

\section{Discussion}

The pulmonary vein (PV) ablation in patients with symptomatic paroxysmal atrial fibrillation (AF) has received increasing attention over the last years and is now a well-established therapeutic approach to limit symptomatic paroxysmal AF [6,7]. The use of novel catheter techniques gives reasons to expect better safety and efficacy, but there is little clinical evidence so far $[16,17]$. Performing MACPAF, we hypothesize that the cryoenergy device (Arctic Front ${ }^{\circ}$ ) is superior to the balloon based device using radiofrequency (HD Mesh ablator ${ }^{\circ}$ ) with regard to efficacy and safety. Efficacy of LACA will be assessed by achieving a complete $\mathrm{PV}$ isolation as an acute effect, and by a lack of AF recurrence after a blanking period of three months (long-term efficacy), evaluated by an implantable device able to detect AF.

Focusing on the safety of LACA, the use of diffusionweighted magnetic resonance imaging will precisely detect (silent) cerebral infarction, as recently demonstrated using a $1.5 \mathrm{~T}$ MRI [15]. The use of a 3T MRI 
enables a higher resolution, improving the significance of stroke detection, while the detection of cerebral hemorrhage, chronic lesions and brain vessel occlusion is at least equal to 1.5T MRI [26]. Therefore, we expect a higher rate of silent strokes than the reported 10$11.3 \%[14,15]$. Furthermore, the MACPAF study will demonstrate if the incidence of clinically silent or clinically evident strokes differs within the tested ablation systems. Moreover, a retrospective analysis of MACPAF might identify LACA procedure-independent risk factors, hopefully reducing LACA-associated stroke rate according to optimized technical conditions and patient selection. Using an implantable device for AF detection and a follow-up MRI after 6 months, we will be able to correlate the rate of (silent) strokes with the AF burden in patients on oral anticoagulation.

According to a http://www.clinicaltrial.org search, the MACPAF study meets highest technical standards. To eliminate potential conflicts of interest, this is an investigator-initiated clinical trial.

Nevertheless, the MACPAF study has its strengths and limitations. Firstly, using two balloon based ablation systems, our results are not transferable to other LACA procedures. Secondly, focusing on paroxysmal AF, we are unable to address questions regarding persistent AF. Thirdly, the assessment of the predefined endpoints is blinded but the LACA procedure itself can not be done in a blinded fashion. Fourth, the limited number of patients might not allow correction for multiple testing. Fifth, according to the study design, a placebo-arm is not intended. The lack of a placebo arm is justified as ablation is a clinically indicated procedure and thus withholding any ablation would be unethical. Prospective multicenter studies will be needed to clarify these important issues.

Taken together, MACPAF is a prospective randomized single-center trial to compare the efficacy and safety of LACA for paroxysmal AF using two balloon based catheter techniques. Results from MACPAF will provide important information helping cardiologists and neurologists to better counsel AF-patients concerning risks and benefits of LACA. Doing MACPAF, we hope to make a significant contribution to the evaluation of the LACA-associated (silent) stroke risk, setting the stage for stroke-risk reduction by improved technical conditions and appropriate patient selection.

\section{List of abbreviations}

AF: atrial fibrillation; LACA: left atrial catheter ablation; MRI: magnetic resonance imaging; MACPAF: Mesh Ablator versus Cryoballoon Pulmonary Vein Ablation of Symptomatic Paroxysmal Atrial Fibrillation Study; PV: pulmonary veins.

\section{Acknowledgements}

We thank Dr. Ute Kopp (Department of Neurology, Charité - University Medicine Berlin, Germany) for her advice regarding neuropsychological tests. The project has received funding from the Federal Ministry of Education and Research via the grant Center for Stroke Research Berlin (01 EO 0801). ME receives support from the Volkswagen-Stiftung, Deutsche Forschungsgemeinschaft and the Federal Ministry of Education and Research.

\section{Author details}

${ }^{1}$ Department of Neurology, Charité - University Medicine Berlin, Germany. ${ }^{2}$ Center for Stroke Research, Charité, Berlin, Germany. ${ }^{3}$ Department of Cardiology and Pneumology, Charité - University Medicine Berlin, Germany.

\section{Authors' contributions}

All authors have read and approved the final manuscript. LK, AS, KGH, ME, HPS and JBF initiated the study. LK and KGH wrote the study-protocol and the manuscript. LK will do the patient inclusion, JU the neuropsychological examination and $\mathrm{KGH}$ the neurological and the MRI examinations under supervision of JBF. Blinded MRI reading will be done by JBF. PUH did and will do the statistical calculations.

\section{Competing interests}

KGH reports lecture fees by Sanofi-Aventis and LK reports lecture fees by Medtronic. ME reports receiving consulting, lecture, grant and/or advisory board fees by BMS, Sanofi-Aventis, Boehringer-Ingelheim, Novartis, Pfizer and AstraZeneca. AS reports lecture fees and prior study grants by Medtronic and C.R. Bard. JBF reports receiving consulting, lecture and advisory board fees by BMS, Siemens, Philips, Perceptive, Bio-Imaging Technologies, Boehringer Ingelheim, Lundbeck and Sygnis.

Received: 4 July 2010 Accepted: 21 July 2010 Published: 21 July 2010

\section{References}

1. Kolominsky-Rabas PL, Weber M, Gefeller O, Neundoerfer B, Heuschmann PU: Epidemiology of ischemic stroke subtypes according to TOAST criteria: incidence, recurrence, and long-term survival in ischemic stroke subtypes: a population-based study. Stroke 2001, 32:2735-2740.

2. Montaner J, Perea-Gainza M, Delgado P, Ribo M, Chacon P, Rosell A, Quintana M, Palacios ME, Molina CA, Alvarez-Sabin J: Etiologic diagnosis of ischemic stroke subtypes with plasma biomarkers. Stroke 2008, 39:2280-2287.

3. Go AS, Hylek EM, Phillips KA, Chang Y, Henault LE, Selby JV, Singer DE: Prevalence of diagnosed atrial fibrillation in adults: national implications for rhythm management and stroke prevention: the AnTicoagulation and Risk Factors in Atrial Fibrillation (ATRIA) Study. JAMA 2001, 285:2370-2375

4. Heeringa J, van der Kuip DA, Hofman A, Kors JA, van Herpen G, Stricker BH, Stijnen T, Lip GY, Witteman JC: Prevalence, incidence and lifetime risk of atrial fibrillation: the Rotterdam study. Eur Heart J 2006, 27:949-953.

5. Marini C, De Santis F, Sacco S, Russo T, Olivieri L, Totaro R, Carolei A: Contribution of atrial fibrillation to incidence and outcome of ischemic stroke: results from a population-based study. Stroke 2005, 36:1115-1119.

6. Fuster V, Ryden LE, Cannom DS, Crijns HJ, Curtis AB, Ellenbogen KA, Halperin JL, Le Heuzey JY, Kay GN, Lowe JE, et al: ACC/AHA/ESC 2006 guidelines for the management of patients with atrial fibrillation: full text: a report of the American College of Cardiology/American Heart Association Task Force on practice guidelines and the European Society of Cardiology Committee for Practice Guidelines (Writing Committee to Revise the 2001 guidelines for the management of patients with atrial fibrillation) developed in collaboration with the European Heart Rhythm Association and the Heart Rhythm Society. Europace 2006, 8:651-745.

7. Chugh A, Morady F: Atrial fibrillation: catheter ablation. J Interv Card Electrophysiol 2006, 16:15-26.

8. Lee SH, Tai CT, Hsieh MH, Tsai CF, Lin YK, Tsao HM, Yu WC, Huang JL, Ueng KC, Cheng JJ, et al: Predictors of early and late recurrence of atrial fibrillation after catheter ablation of paroxysmal atrial fibrillation. J Interv Card Electrophysiol 2004, 10:221-226.

9. Cappato R, Calkins H, Chen SA, Davies W, lesaka Y, Kalman J, Kim YH, Klein G, Natale A, Packer D, et al: Updated worldwide survey on the 
methods, efficacy, and safety of catheter ablation for human atrial fibrillation. Circ Arrhythm Electrophysiol 2010, 3:32-38.

10. Dagres N, Hindricks G, Kottkamp H, Sommer P, Gaspar T, Bode K, Arya A, Husser D, Rallidis LS, Kremastinos DT, Piorkowski C: Complications of atrial fibrillation ablation in a high-volume center in 1,000 procedures: still cause for concern? J Cardiovasc Electrophysiol 2009, 20:1014-1019.

11. Spragg DD, Dalal D, Cheema A, Scherr D, Chilukuri K, Cheng A, Henrikson CA, Marine JE, Berger RD, Dong J, Calkins H: Complications of catheter ablation for atrial fibrillation: incidence and predictors. $J$ Cardiovasc Electrophysiol 2008, 19:627-631.

12. Bertaglia E, Brandolino G, Zoppo F, Zerbo F, Pascotto P: Integration of three-dimensional left atrial magnetic resonance images into a real-time electroanatomic mapping system: validation of a registration method. Pacing Clin Electrophysiol 2008, 31:273-282.

13. van Walraven $C$, Hart RG, Wells GA, Petersen P, Koudstaal PJ, Gullov AL, Hellemons BS, Koefed BG, Laupacis A: A clinical prediction rule to identify patients with atrial fibrillation and a low risk for stroke while taking aspirin. Arch Intern Med 2003, 163:936-943.

14. Lickfett L, Hackenbroch M, Lewalter T, Selbach S, Schwab JO, Yang A, Balta O, Schrickel J, Bitzen A, Luderitz B, Sommer T: Cerebral diffusionweighted magnetic resonance imaging: a tool to monitor the thrombogenicity of left atrial catheter ablation. J Cardiovasc Electrophysiol 2006, 17:1-7.

15. Schrickel JW, Lickfett L, Lewalter T, Mittman-Braun E, Selbach S, Strach K, Nahle CP, Schwab JO, Linhart M, Andrie R, et al: Incidence and predictors of silent cerebral embolism during pulmonary vein catheter ablation for atrial fibrillation. Europace 2010, 12:52-57.

16. Klein G, Oswald H, Gardiwal A, Lusebrink U, Lissel C, Yu H, Drexler H: Efficacy of pulmonary vein isolation by cryoballoon ablation in patients with paroxysmal atrial fibrillation. Heart Rhythm 2008, 5:802-806.

17. Mansour M, Forleo GB, Pappalardo A, Heist EK, Avella A, Laurenzi F, De Girolamo P, Bencardino G, Dello Russo A, Mantica M, et al: Initial experience with the Mesh catheter for pulmonary vein isolation in patients with paroxysmal atrial fibrillation. Heart Rhythm 2008, 5:1510-1516.

18. Hindricks G, Piorkowski C, Tanner H, Kobza R, Gerds-Li JH, Carbucicchio C, Kottkamp H: Perception of atrial fibrillation before and after radiofrequency catheter ablation: relevance of asymptomatic arrhythmia recurrence. Circulation 2005, 112:307-313.

19. Hotter B, Pittl S, Ebinger M, Oepen G, Jegzentis K, Kudo K, Rozanski M, Schmidt WU, Brunecker $P, X u C$, et al: Prospective study on the mismatch concept in acute stroke patients within the first $24 \mathrm{~h}$ after symptom onset - 1000Plus study. BMC Neurol 2009, 9:60,

20. Brott T, Adams HP Jr, Olinger CP, Marler JR, Barsan WG, Biller J, Spilker J, Holleran $R$, Eberle R, Hertzberg $V$, et al: Measurements of acute cerebral infarction: a clinical examination scale. Stroke 1989, 20:864-870.

21. van Swieten JC, Koudstaal PJ, Visser MC, Schouten HJ, van Gijn J: Interobserver agreement for the assessment of handicap in stroke patients. Stroke 1988, 19:604-607.

22. Lezak MD: Neuropsychological Assessment New York: Oxford University Press, 42004.

23. Sturm W, Wilmes K, Horn W: Leistungsprüfsystem für 50-90jährige (LPS 50+) Göttingen: Hogrefe 1993

24. Helmstaedter C, Durwen HF: [The Verbal Learning and Retention Test. A useful and differentiated tool in evaluating verbal memory performance]. Schweiz Arch Neurol Psychiatr 1990, 141:21-30.

25. Lehrl S, Merz J, Erzigkeit H, Galster V: [MWT-A-a repeatable intelligence short-test, fairly independent from psycho-mental disorders]. Nervenarzt 1974, 45:364-369.

26. Frayne R, Goodyear BG, Dickhoff P, Lauzon ML, Sevick RJ: Magnetic resonance imaging at 3.0 Tesla: challenges and advantages in clinical neurological imaging. Invest Radiol 2003, 38:385-402.

\section{Pre-publication history}

The pre-publication history for this paper can be accessed here: http://www.biomedcentral.com/1471-2377/10/63/prepub

doi:10.1186/1471-2377-10-63

Cite this article as: Haeusler et al: Stroke risk associated with balloon based catheter ablation for atrial fibrillation: Rationale and design of the MACPAF Study. BMC Neurology 2010 10:63.

\section{Submit your next manuscript to BioMed Central and take full advantage of:}

- Convenient online submission

- Thorough peer review

- No space constraints or color figure charges

- Immediate publication on acceptance

- Inclusion in PubMed, CAS, Scopus and Google Scholar

- Research which is freely available for redistribution 